\title{
The validity degree vectors of formulae in two-valued predicate logic
}

\author{
Xiaoyan Qin ${ }^{1,2}$ Yang Xu $^{1}$ Yi Liu ${ }^{1}$ \\ ${ }^{1}$ Intelligent Control Development Center, Southwest Jiaotong University, \\ NO.111 of the North Second Ring Road, \\ Chengdu, Sichuan Province 610031, P.R.China \\ E-mail: lisaqin1981@126.com(X.Y. Qin); xuyang@home.swtju.edu.cn(Y.Xu) \\ ${ }^{2}$ College of Mathematics and Computer Science, Shanxi Normal University, \\ No. 1 of Gongyuan Road, \\ Linfen, Shanxi Province 041004, P.R.China
}

Received 14 April 2014

Accepted 1 June 2015

\begin{abstract}
By means of infinite product of uniformly distributed probability spaces of cardinal $n$, the concept of $n$-validity degrees and validity degree vectors of formulae in two-valued predicate logic are introduced. It is proved that the validity degree vectors of formulae can preserve the logical relation between formulae. Moreover, a consistency theorem is obtained which says that the $n$-validity degree $\tau_{n}(A)$ of the quantifierfree first-order formula $A$ without any repeated predicate symbols or terms is independent of the natural number $n$, and is a constant equal to the validity degree $\tau\left(A^{0}\right)$ of the corresponding proposition $A^{0}$ in classical propositional logic.
\end{abstract}

Keywords: two-valued predicate logic, $n$-validity degree, validity degree vector, consistency theorem

\section{Introduction}

There are a variety of statements in the real world, and people's judgements about the truth of these statements are various: true, false, basically true, almost true, and etc. However, in the classical propositional logic(see, e.g., [1]), there are only the notions of tautologies and contradictions for abstract symbolized propositions, which respectively indicate the absolutely true and absolutely false propositions. In some ways, it's a flaw that the truth degrees of most of the formulae, which are neither tautologies nor contradictions, weren't discussed. In fact, the basic idea of universal validity degrees of logic formulae has long been a concern of diverse logicians. In 1952, Rosser and Turquette proposed the idea of distinguishing reliabilities among formulae in many-valued logic ${ }^{[2]}$, and this idea was discussed and developed by many scholars from different points of view (see, e.g., refs. [3-7]). In 2001, Wang ${ }^{[8]}$ proposed the definition of universal validity degrees of general propositions in classical twovalued propositional logic, using the countable infinite product of a family of evenly distributed probability measure space with their cardinality of 2 . It initiated a series of subsequent researches (see refs. [9-27]). Now a unified and fairly complete theory of quantitative logics has been formed in diverse multiple valued propositional logics (see refs. [28,29]).

Nevertheless, it's worth noting that the above researches are all in the propositional logic. The expression ability of predicate logic is more powerful 
than the one of the propositional logic, so we naturally hope to establish the theory of truth degrees of formulae in the former. But, because of the complexity of interpretations for first-order language, it's quite hard to complete this work in predicate logic, and so far there are only some sporadic results such as refs. [18,30-40]. Among them, the work in ref. [18] is just a preparation for introducing more general truth degree theory in predicate logic, and only the truth degrees of binary first-order formulae can be gained indirectly because modal logic is essentially a segment of predicate logic as pointed out in ref. [41]. Moreover, in the early of 1980, Bandler and Kohout proposed and developed the theory of checklist paradigm in their series of papers [30-35], where they gave a kind of method for calculating validity degrees of formulae in a wider sense. However, besides the proposition formulae, they only considered the unary and binary first-order formulae. Distinct to the former two, ref. [36] first established an axiomatic theory of truth degree of formulae from the point view of syntactic instead of semantics, but it's a pity that only the truth degrees of all the closed formulae in predicate logic was studied. Compared with the former three, the model proposed in ref. [37-40] presented a general theory of universal validity degrees of all first-order formulae, but it still was only a preliminary test far from satisfactory because it defined validity degrees for predicate formulae simply by taking the average of the values obtained from two extreme cases.

The present paper will express the validity degrees of first-order formulae in the form of vector for the first time, and take the average value of the relative satisfiability degrees of the first-order formula in all possible finite interpretations instead of only two extreme cases. Moreover, the calculation in this paper is far more complex than the corresponding calculation in classical propositional logic, because the valuations on the variables in a first-order formula can be any subset of the domain of a finite interpretation, while that of the latter only need to be chosen from the two-element-set of $\{0,1\}$. However, it is interesting that a consistency theorem is obtained in the present paper, which says that for any given quantifier-free formula $A$ without any re- peated predicate symbols or terms in first-order logic and for every natural number $n$ the $n$-validity degree $\tau_{n}(A)$ is independent of the number $n$, and is a constant equal to the validity degree $\tau\left(A^{0}\right)$ of the corresponding proposition $A^{0}$ in classical propositional logic given in ref. [8]. Therefore, the consistency theorem provides a convenient way to calculate the validity degree vector of a big class of first-order formulae, that is, we only need to calculate the validity degree of the corresponding proposition in classical propositional logic and it is much easier.

In the present paper, some necessary preliminaries about the validity degrees of propositions and first-order formulae are introduced in section 2 . The concept of $n$-validity degree and validity degree vector of a first-order formula are proposed in section 3 and basic properties are discussed. In section 4 , the consistency theorem is proved so that we obtain a convenient way to calculate the validity degree vectors of a big class of formulae in two-valued predicate logic. Finally, we will introduce some following work in section 5 .

\section{Preliminaries}

\subsection{The degree of universal validity of a proposition}

Assume that $S=\left\{p_{1}, p_{2}, \cdots\right\}$ is a countable set, the members of $S$ are called atomic propositional formulae. Let $F(S)$ be the free algebra of type $(1,2)$ generated by $S$, where $\neg$ is the unary operator, and $\rightarrow$ is the binary operator, i.e., $S \subset F(S)$ and $A, B \in F(S)$ imply that $\neg A$ and $A \rightarrow B \in F(S)$, and $F(S)$ contains no other members. Define on $\{0,1\}$ a unary operator $\neg$ and a binary operator $\rightarrow$ as follows:

$$
\begin{gathered}
\neg 1=0, \neg 0=1, \\
a \rightarrow b=0 \text { iff } a=1 \text { and } b=0, a, b \in\{0,1\}
\end{gathered}
$$

then $\{0,1\}$ becomes an algebra of type $(1,2)$. A mapping $v: F(S) \rightarrow\{0,1\}$ is said to be a valuation of $F(S)$ if $v$ is a homomorphism of type $(1,2)$, i.e.,

$$
v(\neg A)=\neg v(A), \quad v(A \rightarrow B)=v(A) \rightarrow v(B),
$$

where $A, B \in F(S)$. The set consisting of all valuations of $F(S)$ will be denoted by $\Omega$. It is well known that every valuation $v$ of $F(S)$ is completely 
determined by its restriction $v \mid S: S \rightarrow\{0,1\}$ because $F(S)$ is the free algebra generated by $S^{[42]}$. Suppose that $\left(X_{n}, \mathscr{A}_{n}, \mu_{n}\right)$ is a probabilistic measure space, where $\mu_{n}$ is a probability measure on $X_{n}$, and $\mathscr{A}_{n}$ is the family consisting of all $\mu_{n}$-measurable subsets of $X_{n}(n=1,2, \cdots)$. Assume that $X=\prod_{n=1}^{\infty} X_{n}$, then $\prod_{n=1}^{\infty} \mathscr{A}_{n}$ generates on $X$ a $\sigma$-algebra $\mathscr{A}$, and there exists on $X$ a unique measure $\mu$ such that (i) $\mathscr{A}$ is the family of all $\mu$-measurable subsets of $X$; (ii) $E \times \prod_{n=m+1}^{\infty} X_{n}$ is $\mu$-measurable and

$\mu\left(E \times \prod_{n=m+1}^{\infty} X_{n}\right)=\left(\mu_{1} \times \mu_{2} \times \cdots \times \mu_{m}\right)(E), m=1,2, \cdots$,

whenever $E$ is a measurable subset of $\prod_{n=1}^{m} X_{n} . \mu$ is called the infinite product of $\mu_{1}, \mu_{2}, \cdots$ (see [43, Theorem B, p157]). The probability measure space $(X, \mathscr{A}, \mu)$ will often be simplified as $\mathbf{X}$. We assume in the present paper that

$$
X_{1}=X_{2}=\cdots=M=\left\{m_{1}, m_{2}, \cdots, m_{|M|}\right\},
$$

where $|M|$ is the cardinality of the finite set $M$, and

$$
\mu_{n}\left(\left\{m_{1}\right\}\right)=\mu_{n}\left(\left\{m_{2}\right\}\right)=\cdots=\mu_{n}\left(\left\{m_{|M|}\right\}\right)=\frac{1}{|M|} .
$$

The concept of degree of universal validity of propositional formulae was defined in [8] as follows: suppose that $M=\{0,1\}$ in (3), and $v\left(p_{n}\right)=v_{n}(n=$ $1,2, \cdots)$, then $\vec{v}=\left(v_{1}, v_{2}, \cdots\right) \in X=\{0,1\}^{\infty}$. Conversely, assume that $\vec{v}=\left(v_{1}, v_{2}, \cdots\right) \in X$, then there exists a unique $v \in \Omega$ such that $v\left(p_{n}\right)=v_{n}(n=$ $1,2, \cdots)$. Hence there is a bijection $\varphi: \Omega \rightarrow X$ such that $\varphi(v)=\vec{v}, \varphi$ is said to be the measurable mapping of $\Omega$. Suppose that $A \in F(S)$, let

$$
[A]=\{\vec{v} \in X \mid v \in \Omega, v(A)=1\}, \tau(A)=\mu([A]),
$$

then $\tau(A)$ is called the degree of universal validity of $A^{[8]}$.

\subsection{Basic concepts and symbols used in the present paper}

A first order language $\mathscr{L}$ has the following as its alphabet of symbols: (i) variables $x_{1}, x_{2}, \cdots$; (ii) some individual constants $c_{1}, c_{2}, \cdots$; (iii) some predicate letters $P, Q, R, \cdots$; (iv) some function letters $f, g, h, \cdots ;$ (v) the punctuation symbols"(",")" and ","; (vi) the connectives $\neg$ and $\rightarrow$; (vii) the universal quantifier $\forall$; (viii) the existential quantifier $\exists$, which is an abbreviation of $\neg \forall \neg$ (see, e.g. [6]). In the present paper, we agree on the assumption that there is no function symbols in the alphabet, which is adopted in, for example, refs.[3,42] etc. Then $x_{1}, x_{2}, \cdots$ and $c_{1}, c_{2}, \cdots$ are called terms. An atomic formula has the form $P\left(t_{1}, \cdots, t_{k}\right)$ where $P$ is a predicate letter of arity $k$ and $t_{1}, \cdots, t_{k}$ are terms. A well-formed formula (briefly wff or first-order formula, or simply formula) of $\mathscr{L}$ is defined by:(i) Each atomic formula of $\mathscr{L}$ is a formula. (ii) If $A$ and $B$ are formulae of $\mathscr{L}$, then so are $\neg A, A \rightarrow B$ and $\left(\forall x_{i}\right) A$. (iii) The set of formulae of $\mathscr{L}$ is generated as in (i) and (ii). The set of all formulae of $\mathscr{L}$ will be denoted by $\mathscr{F}$.

An interpretation $\mathbf{M}=\left(M,\left(r_{P}\right)_{P},\left(m_{c}\right)_{c}\right)($ or, $\{0,1\}$ structure called by Hájek in [3]) is a triplet where $M$ is a nonempty set, called the domain of $\mathbf{M}$, for each predicate letter $P$ of arity $k, r_{P}$ is a relation of arity $k$ on $M$, i.e., $r_{P} \subset M^{k}$, and for each individual constant $c$, there exists a unique corresponding element $m_{c}$ in $M$. A valuation $v$ of $\mathscr{L}$ in $\mathbf{M}$ is a mapping from the set $\mathscr{T}$ of terms to $M$ satisfying $v(c)=m_{c}$ and $v\left(x_{n}\right) \in M(n=1,2, \cdots)$. A valuation $v$ in $\mathbf{M}$ is said to satisfying a formula $A \in \mathscr{F}$ if it can be shown inductively to do so under the following four conditions: (i) $v$ satisfies the atomic formula $P\left(t_{1}, \cdot, t_{k}\right)$ if $r_{P}\left(v\left(t_{1}\right), \cdots, v\left(t_{k}\right)\right)$ is true in M, i.e., $\left(v\left(t_{1}\right), \cdots, v\left(t_{k}\right)\right) \in r_{P}$. (ii) $v$ satisfies $\neg B$ if $v$ does not satisfy $B$. (iii) $v$ satisfies $B \rightarrow C$ if either $v$ satisfies $\neg B$ or $v$ satisfies $C$. (iv) $v$ satisfies $\left(\forall x_{i}\right) B$ if every valuation $v^{\prime}$ which is $i$-equivalent to $v$ satisfies $B$, where $v^{\prime}$ is $i$-equivalent to $v$ iff $v^{\prime}\left(x_{n}\right)=v\left(x_{n}\right)$ whenever $n \neq i$. The set of all valuations in $\mathbf{M}$ will be denoted by $\Omega_{\mathbf{M}}$. Assume that $A \in \mathscr{F}$ and $v \in \Omega_{\mathbf{M}}$, then we use $\|A\|_{\mathbf{M}, v}=1$ to denote that $v$ satisfies $A$ and use $\|A\|_{\mathbf{M}, v}=0$ to denote that $v$ does not satisfy $A$. If $\|A\|_{\mathbf{M}, v}=1$ holds 
for every valuation $v \in \Omega_{\mathbf{M}}$, then we say that $\mathbf{M}$ is a model of $A$, or $A$ is true in $\mathbf{M}$, and denoted by $\|A\|_{\mathbf{M}}=1$. If every interpretation $\mathbf{M}$ is a model of $A$, then we say that $A$ is logically valid.

Let $A \in \mathscr{F}$. $A$ is said to be in disjunctive normal form and in conjunctive normal form, respectively, if $A$ has the respective forms

$$
\left(Q_{11} \wedge \cdots \wedge Q_{1 n}\right) \vee \cdots \vee\left(Q_{m 1} \wedge \cdots \wedge Q_{m n}\right),
$$

and

$$
\left(Q_{11} \vee \cdots \vee Q_{1 n}\right) \wedge \cdots \wedge\left(Q_{m 1} \vee \cdots \vee Q_{m n}\right),
$$

where each $Q_{i j}=p_{j}$ or $Q_{i j}=\neg p_{j}(i=$ $1,2, \cdots, m ; j=1,2, \cdots, n)$, each $p_{j}$ is an atomic formula in $\mathscr{F}$.

$A$ is said to be in prenex normal form if it is of the form

$$
\left(Q_{1} x_{i_{1}}\right) \cdots\left(Q_{m} x_{i_{m}}\right) D, m \geqslant 0,
$$

where each $Q_{j}$ is either the universal quantifier $\forall$ or the existential quantifier $\exists$, and $D$ is quantifier-free.

\subsection{The average validity degree of a first-order formula}

As we know, propositional logic is decidable, i.e., whether a formula $A$ is a tautology or not can be verified by certain algorithm, but predicate logic is undecidable, i.e., there is no algorithm for deciding whether or not a given first-order formula $A$ is logically valid. It is chiefly because the class of all the interpretations of a first-order language is beyond the category of the theory of sets. This situation motivates the study of finite interpretations(i.e., interpretations with finite domains) and finite models such as ref.[18],[31], and etc. Note that it may happen that every finite interpretation is a model of a formula $A$ while $A$ is not logically valid, a famous example is given by Hilbert as follows ${ }^{[44]}$

$$
\begin{gathered}
A^{*}=(\exists x) P(x, x) \vee(\exists x)(\exists y)(\exists z)(P(x, y) \wedge P(y, z) \\
\wedge \neg P(x, z)) \vee(\exists x)(\forall y) \neg P(y, x) .
\end{gathered}
$$

However, it is clear that interpretations with finite domains are easy to be handled and a first-order formula $A$ can certainly be considered to be good to some extent if $A$ is true in every finite interpretation. And in what follows we consider only finite interpretations, and we use the symbol $\mathscr{M}_{f}$ to denote the class of all finite interpretations.

Suppose that $\left(X_{n}, \mathscr{A}_{n}, \mu_{n}\right)$ is a sequence of totally finite measure spaces with $\mu_{n}\left(X_{n}\right)=1$, and $(X, \mathscr{A}, \mu)$ is the product probability measure space mentioned in Section 2.1, where $X_{n}$ and $\mu_{n}$ are defined by (3) and (4),respectively. In what follows the product space $(X, \mathscr{A}, \mu)$ will be simplified as $X_{M}$. Assume that $v: \mathscr{T} \rightarrow M$ is a valuation of the first-order language $\mathscr{L}$ in the finite interpretation $\mathbf{M}=\left(M,\left(r_{P}\right)_{P},\left(m_{c}\right)_{c}\right)$, since $v(c)=m_{c}$ is already fixed for every individual constant $c, v$ is completely determined by its restriction $v \mid W$ on the set $W=\left\{x_{1}, x_{2}, \cdots\right\}$ of variables. Assume that $v\left(x_{k}\right)=$ $v_{k}(k=1,2, \cdots)$, then $\vec{v}=\left(v_{1}, v_{2}, \cdots\right) \in X_{M}$. Conversely, assume that $\vec{v}=\left(v_{1}, v_{2}, \cdots\right) \in X_{M}$, then there exists a unique $v \in \Omega_{\mathbf{M}}$ such that $v\left(x_{k}\right)=$ $v_{k}(k=1,2, \cdots)$. Hence $\varphi_{\mathbf{M}}: \Omega_{\mathbf{M}} \rightarrow X_{M}$ is a bijection where $\varphi_{\mathbf{M}}(v)=\vec{v} \in X_{M}$.

Definition 1. ${ }^{[30]}$ Let $\mathbf{M} \in \mathscr{M}_{f}$ and $A \in \mathscr{F}$. Define $[A]_{\mathbf{M}}$ and $\tau_{\mathbf{M}}(A)$ as follows respectively

$$
\begin{gathered}
{[A]_{\mathbf{M}}=\left\{\vec{v} \in X_{M} \mid v \in \Omega_{\mathbf{M}},\|A\|_{\mathbf{M}, v}=1\right\},} \\
\tau_{\mathbf{M}}(A)=\mu_{\mathbf{M}}\left([A]_{\mathbf{M}}\right),
\end{gathered}
$$

then $\tau_{\mathbf{M}}(A)$ is called the relative satisfiability degree of $A$ in $\mathbf{M}$.

Example 1. Let $A=P(x) \rightarrow(\forall x) P(x) \in \mathscr{F}$, $\mathbf{M}=\left(M,\left\{r_{P}\right\},\left(m_{c}\right)_{c}\right) \in \mathscr{M}_{f}$. Since only one variable $x$ appears in the formulae $A, v(y)(y$ is distinct from $x$ ) can be obviated, hence we consider $M=\{v(x) \mid v(x) \in M\}$ instead of $X_{M}$. It follows from Definition 1 that

$$
\begin{aligned}
\tau_{\mathbf{M}}(A)= & \mu_{\mathbf{M}}\left(\left\{\vec{v} \in X_{M} \mid v \in \Omega_{\mathbf{M}},\right.\right. \\
& \left.\left.\|P(x) \rightarrow(\forall x) P(x)\|_{\mathbf{M}, v}=1\right\}\right) \\
= & 1-\mu_{\mathbf{M}}\left(\left\{\vec{v} \in X_{M} \mid v \in \Omega_{\mathbf{M}},\right.\right. \\
& \left.\left.\|P(x) \rightarrow(\forall x) P(x)\|_{\mathbf{M}, v}=0\right\}\right) \\
= & 1-\mu_{\mathbf{M}}\left(\left\{\vec{v} \in X_{M} \mid v \in \Omega_{\mathbf{M}},\right.\right. \\
& \left.\left.\|P(x)\|_{\mathbf{M}, v}=1,\|(\forall x) P(x)\|_{\mathbf{M}, v}=0\right\}\right) .
\end{aligned}
$$

Thus, it's obvious that $\tau_{\mathbf{M}}(A)=1$ holds whenever $r_{P}=M$ or $r_{P}=\emptyset$. Otherwise, for every valuation $v$ in $\Omega_{\mathbf{M}}$, we have that $\|(\forall x) P(x)\|_{\mathbf{M}, v}=0$. Then it follows the above equation that $\tau_{\mathbf{M}}(A)=1-\frac{\left|r_{P}\right|}{|M|}$. 
Consequently, we have that

$\tau_{\mathbf{M}}(A)=\left\{\begin{array}{cl}1 & , \text { if } r_{P}=\emptyset, \text { or } r_{P}=M, \\ 1-\frac{\left|r_{P}\right|}{|M|} \quad, & \text { else. } \square\end{array}\right.$

Proposition 1. ${ }^{[30]}$ Suppose that $A \in \mathscr{F}, A$ is a prenex normal form. If A contains no quantifiers, or the quantifiers in A are all universal or all existential, then

(i) A is logically valid if and only if for every $\mathbf{M} \in \mathscr{M}_{f}, \tau_{\mathbf{M}}(A)=1$;

(ii) $A$ is a contradiction if and only if for every $\mathbf{M} \in \mathscr{M}_{f}, \tau_{\mathbf{M}}(A)=0$.

Definition 2. ${ }^{[30]}$ Let $A \in \mathscr{F}$. Define $\bar{\tau}(A)$ as follows:

$\bar{\tau}(A)=\frac{\sup \left\{\tau_{\mathbf{M}}(A) \mid \mathbf{M} \in \mathscr{M}_{f}\right\}+\inf \left\{\tau_{\mathbf{M}}(A) \mid \mathbf{M} \in \mathscr{M}_{f}\right\}}{2}$

then $\bar{\tau}(A)$ is called the average validity degree of $A$.

Example 2. Suppose $A=P(x) \rightarrow(\forall x) P(x) \in \mathscr{F}$ . Let $\mathbf{M}_{\mathbf{n}}=\left(M_{n},\left\{r_{P}\right\},\left(m_{c}\right)_{c}\right) \in \mathscr{M}_{f}$, where $\left|M_{n}\right|=$ $n,\left|r_{P}\right|=n-1(n=1,2, \cdots)$. It follows from Example 1 that

$$
\tau_{\mathbf{M}_{\mathbf{n}}}(A)=1-\frac{n-1}{n}=\frac{1}{n} .
$$

It follows that

$$
\inf \left\{\tau_{\mathbf{M}_{\mathbf{n}}}(A) \mid \mathbf{M}_{\mathbf{n}} \in \mathscr{M}_{f}, n=1,2, \cdots\right\}=0,
$$

and then

$$
\inf \left\{\tau_{\mathbf{M}}(A) \mid \mathbf{M} \in \mathscr{M}_{f}\right\}=0 .
$$

Moreover, let $\mathbf{M}=\left(M,\left\{r_{P}\right\},\left(m_{c}\right)_{c}\right) \in \mathscr{M}_{f}$, where $r_{P}=M$, then it follows from Example 1 that $\tau_{\mathbf{M}}(A)=1$. Furthermore

$$
\sup \left\{\tau_{\mathbf{M}}(A) \mid \mathbf{M} \in \mathscr{M}_{f}\right\}=1 .
$$

According to (8),(9) and (10), it is clear that $\bar{\tau}(A)=\frac{1}{2}$.

According to (8), it is easy to prove that

Proposition 2. Suppose $A \in \mathscr{F}$, then $\bar{\tau}(A)=1$ iff $\tau_{\mathbf{M}}(A)=1$ holds for every interpretation $\mathbf{M} \in \mathscr{M}_{f}$, and $\bar{\tau}(A)=0$ iff $\tau_{\mathbf{M}}(A)=0$ holds for every interpretation $\mathbf{M} \in \mathscr{M}_{f}$.

\section{The validity degree vector of a first-order formula}

The work about the average validity degrees of firstorder formulae is only a preliminary test far from satisfactory because it defined truth degrees by taking the average of the values obtained from two extreme cases. Take a simple example, suppose $B=$ $Q(x, c), C=(\exists x) Q(x, c), D=(\forall x)(P(x) \rightarrow R(x))$. Let $\mathbf{M}_{1}=\left(M_{1},\left\{r_{P}, r_{Q}, r_{R}\right\},\left\{m_{c}\right\}\right)$, where $r_{P}=r_{Q}=$ $M_{1}$ and $r_{R}=\emptyset$, and $\mathbf{M}_{2}=\left(M_{2},\left\{r_{P}, r_{Q}, r_{R}\right\},\left\{m_{c}\right\}\right)$, where $r_{P}=r_{Q}=r_{R}=\emptyset$. From (7), it is not difficult to verify that

$$
\tau_{\mathbf{M}_{1}}(B)=1, \tau_{\mathbf{M}_{1}}(C)=1, \tau_{\mathbf{M}_{1}}(D)=0,
$$

and

$$
\tau_{\mathbf{M}_{2}}(B)=0, \tau_{\mathbf{M}_{2}}(C)=0, \tau_{\mathbf{M}_{2}}(D)=1 .
$$

So, from (8) it follows that $\bar{\tau}(B)=\bar{\tau}(C)=\bar{\tau}(D)=\frac{1}{2}$. Along with the formula $A=P(x) \rightarrow(\forall x) P(x)$ in Example 2 , the average validity degrees of these four formulae are all equal to $\frac{1}{2}$. But, are their truth degrees really equivalent? what are the trends of their truth degree respectively with the change of the finite interpretations? In the following, we will propose the concept of the validity degree vector of a first-order formula to answer these questions.

Note that, if the cardinalities of two sets $M$ and $M^{\prime}$ are equal, i.e., there exists a one-toone correspondence $h: M \rightarrow M^{\prime}$ between them, then the interpretation $\mathbf{M}=\left(M,\left(r_{P}\right)_{P},\left(m_{c}\right)_{c}\right)$ can be naturally changed into the interpretation $\mathbf{M}^{\prime}=$ $\left(M^{\prime},\left(r_{P}^{\prime}\right)_{P},\left(m_{c}^{\prime}\right)_{c}\right)$, where, for the $k$-ary predicate $P,\left(x_{1}, x_{2}, \cdots, x_{k}\right) \in r_{P}$ iff $\left(h\left(x_{1}\right), h\left(x_{2}\right), \cdots, h\left(x_{k}\right)\right) \in$ $r_{P}^{\prime}(k=1,2, \cdots)$, and, for the individual constant $c$, $m_{c}^{\prime}=h\left(m_{c}\right)$. For this reason, in the following we will think of those interpretations having the same structures but different carriers as identical ones. Especially, without any loss of generality, we will take the set $M_{n}=\{1,2, \cdots, n\}$ as a standard universe of discourse for all the finite interpretations of which the cardinalities of the domains are all equal to $n$. In the sequel, we denote by $\mathscr{M}_{n}$ the set of all interpretations possessing standard domain $M_{n}$.

It is clear that $M_{n}^{k}(k=1,2, \cdots)$ is also finite if $M_{n}$ is a finite set. Due to $r_{P} \subseteq M_{n}^{k}$ and $m_{c} \in M_{n}$ for each $k$-ary predicate symbol $P$ and each individual 
constant $c$, the amounts of all possible $r_{P}$ and $m_{c}$ are finite. Furthermore the amount of the interpretations with the domain $M_{n}$ is finite for every specific formula, i.e., $\left|\mathscr{M}_{n}\right|$ is a finite number. So the following definition is reasonable.

Definition 3. Let $A \in \mathscr{F}$. For every positive integer $n$, define $\tau_{n}(A)$ as follows:

$$
\tau_{n}(A)=\frac{1}{\left|\mathscr{M}_{n}\right|} \sum\left\{\tau_{\mathbf{M}}(A) \mid \mathbf{M} \in \mathscr{M}_{n}\right\},
$$

then $\tau_{n}(A)$ is called the $n$-validity degree of $A$. Furthermore,

$$
\vec{\tau}(A)=\left(\tau_{1}(A), \tau_{2}(A), \cdots, \tau_{n}(A), \cdots\right),
$$

then $\vec{\tau}(A)$ is called the validity degree vector of $A$.

Suppose that $\vec{\tau}(A)=\left(\tau_{1}(A), \tau_{2}(A), \cdots, \tau_{n}(A), \cdots\right)$, $\vec{\tau}\left(A^{\prime}\right)=\left(\tau_{1}\left(A^{\prime}\right), \tau_{2}\left(A^{\prime}\right), \cdots, \tau_{n}\left(A^{\prime}\right), \cdots\right)$, and $k$ is a constant. In the present paper, we assume that

$$
\begin{gathered}
\vec{\tau}(A) \leqslant \vec{\tau}\left(A^{\prime}\right) \text { iff } \tau_{n}(A) \leqslant \tau_{n}\left(A^{\prime}\right), \forall n \in Z^{+} ; \\
\vec{\tau}(A) \pm \vec{\tau}\left(A^{\prime}\right)=\left(\tau_{1}(A) \pm \tau_{1}\left(A^{\prime}\right), \tau_{2}(A) \pm \tau_{2}\left(A^{\prime}\right)\right. \\
\left.\cdots, \tau_{n}(A) \pm \tau_{n}\left(A^{\prime}\right), \cdots\right) ; \\
k \vec{\tau}(A)=\left(k \tau_{1}(A), k \tau_{2}(A), \cdots, k \tau_{n}(A), \cdots\right)
\end{gathered}
$$

Example 3. Suppose $B=Q(x, c), C=(\exists x) Q(x, c)$. Assume that $\mathbf{M}=\left(M_{n},\left\{r_{Q}\right\},\left\{m_{c}\right\}\right)$, where $M_{n}=$ $\{1,2, \cdots, n\}$. Since $r_{Q} \subset M^{2}, m_{c} \in M$, there are $2^{\left|M^{2}\right|}=2^{n^{2}}$ subsets of $M^{2}$ which can be taken as $r_{Q}$, and $n$ elements of $M$ which can be taken as $m_{c}$. So $\left|\mathscr{M}_{n}\right|=n 2^{n^{2}}$.

(i)Suppose that $m_{c}=i(i=1,2, \cdots, n)$, there are $C_{n}^{k} \cdot 2^{n(n-1)}$ subsets of $M_{n}^{2}$ which contain only $k$ elements like $(x, i)$, where $x \in M_{n}$, moreover $\tau_{\mathbf{M}}(B)=$ $\frac{k}{n}$ at this point. Therefore, according to (11) and $\sum_{k=0}^{n} k C_{n}^{k}=n \cdot 2^{n-1}$,

$$
\begin{aligned}
\tau_{n}(B) & =\frac{1}{\left|\mathscr{M}_{n}\right|} \sum\left\{\tau_{\mathbf{M}}(B) \mid \mathbf{M} \in \mathscr{M}_{n}\right\} \\
& =\frac{1}{n \cdot 2^{n^{2}}} \sum_{i=1}^{n} \sum_{k=0}^{n} C_{n}^{k} \cdot 2^{n(n-1)} \cdot \frac{k}{n} \\
& =\frac{n}{n \cdot 2^{n^{2}}} \cdot \frac{2^{n(n-1)}}{n} \cdot \sum_{k=0}^{n} k C_{n}^{k} \\
& =\frac{n}{n \cdot 2^{n^{2}}} \cdot \frac{2^{n(n-1)}}{n} \cdot n \cdot 2^{n-1} \\
& =\frac{1}{2}
\end{aligned}
$$

In conclusion, $\vec{\tau}(B)=\left(\frac{1}{2}, \frac{1}{2}, \cdots, \frac{1}{2}, \cdots\right)$.

(ii) Suppose that $m_{c}=i(i=1,2, \cdots, n)$, then $\tau_{\mathbf{M}}(C)=0$ if and only if there doesn't exist any element like $(x, c)$ in $r_{Q}$ where $x \in M_{n}$. In order to take $r_{Q}$ containing no elements like $(x, c)$, we just take one as $r_{Q}$ from all the subsets of the set $M_{n}^{2}-\left\{(x, c) \mid x \in M_{n}\right\}$, and the totality of this kind of subsets is $2^{n^{2}-n}$, that is, there are $2^{n^{2}-n}$ possible $r_{Q}$ not containing elements like $(x, c)$. According to $r_{Q} \subset M_{n}^{2}$, there are $2^{n^{2}}-2^{n^{2}-n}$ possible $r_{Q}$ containing elements like $(x, c)$, and $\tau_{\mathbf{M}}(C)=1$ at this point. Therefore

$$
\begin{aligned}
\tau_{n}(C) & =\frac{1}{\left|\mathscr{M}_{n}\right|} \sum\left\{\tau_{\mathbf{M}}(C) \mid \mathbf{M} \in \mathscr{M}_{n}\right\} \\
& =\frac{1}{n \cdot 2^{n^{2}}} \sum_{i=1}^{n}\left[2^{n^{2}-n} \cdot 0+\left(2^{n^{2}}-2^{n^{2}-n}\right) \cdot 1\right] \\
& =\frac{n}{n \cdot 2^{n^{2}}}\left(2^{n^{2}}-2^{n^{2}-n}\right) \\
& =1-\frac{1}{2^{n}} .
\end{aligned}
$$

In conclusion, $\vec{\tau}(C)=\left(\frac{1}{2}, \frac{3}{4}, \cdots, 1-\frac{1}{2^{n}}, \cdots\right)$

Remark 1. Similar to Example 3(i), we can prove that $\tau_{n}(A)=\frac{1}{2}(n=1,2, \cdots)$ holds for every atomic formula $A$, that is, $\vec{\tau}(A)=\left(\frac{1}{2}, \frac{1}{2}, \cdots, \frac{1}{2}, \cdots\right)$.

Example 4. Suppose $A=P(x) \rightarrow(\forall x) P(x), D=$ $(\forall x)(P(x) \rightarrow R(x)) . \quad$ Assume that $\mathbf{M}=$ $\left(M_{n},\left\{r_{P}, r_{R}\right\},\left(m_{c}\right)_{c}\right)$, where $M_{n}=\{1,2, \cdots, n\}$.

(i) We can take no account of $\left(m_{c}\right)_{c}$ since no individual constant occurs in $A$. So, due to $r_{P} \subseteq M_{n}$, we have $\left|\mathscr{M}_{n}\right|=2^{n}$. Furthermore, if $\left|r_{P}\right|=k(k=$ $0,1, \cdots, n-1)$, there are $C_{n}^{k}$ subsets of $M_{n}$ which can be taken as $r_{P}$, and it follows from Example 1 that $\tau_{\mathbf{M}}(A)=1-\frac{k}{n}$ at this point, and $\tau_{\mathbf{M}}(A)=1$ if $r_{P}=M_{n}$. According to (11),

$$
\begin{aligned}
\tau_{n}(A) & =\frac{1}{\left|\mathscr{M}_{n}\right|} \sum\left\{\tau_{\mathbf{M}}(A) \mid \mathbf{M} \in \mathscr{M}_{n}\right\} \\
& =\frac{1}{2^{n}}\left\{1+\sum_{k=0}^{n-1}\left[C_{n}^{k}\left(1-\frac{k}{n}\right)\right]\right\} \\
& =\frac{1}{2^{n}}\left[1+\sum_{k=0}^{n-1} C_{n}^{k}-\sum_{k=0}^{n-1} C_{n}^{k} \frac{k}{n}\right] \\
& =\frac{1}{2^{n}}\left[1+2^{n}-1-\frac{1}{n}\left(n \cdot 2^{n-1}-n\right)\right] \\
& =\frac{1}{2}+\frac{1}{2^{n}} .
\end{aligned}
$$

In conclusion, $\vec{\tau}(A)=\left(1, \frac{3}{4}, \cdots, \frac{1}{2}+\frac{1}{2^{n}}, \cdots\right)$.

(ii) Similarly, we can take no account of $\left(m_{c}\right)_{c}$ since no individual constant occurs in $D$. Due to $r_{P} \subseteq M_{n}$ and $r_{R} \subseteq M_{n}$, there are $2^{n}$ subsets which 
can be taken as $r_{P}$ or $r_{R}$. So, $\left|\mathscr{M}_{n}\right|=2^{n} \cdot 2^{n}=2^{2 n}$. Moreover,

$$
\tau_{\mathbf{M}}(D)=\left\{\begin{array}{lll}
1 & , & \text { if } r_{P} \subseteq r_{R} \\
0, & \text { else. }
\end{array}\right.
$$

Therefore, we need to consider how many pairs of $\left(r_{P}, r_{R}\right)$ satisfy the condition that $r_{P} \subseteq r_{R}$. In fact, when $\left|r_{R}\right|=k(k=0,1, \cdots, n)$, there are $2^{k}$ subsets of $r_{R}$ which can be chosen as $r_{P}$. And there are $C_{n}^{k}$ subsets of $M_{n}$ which can be taken as $r_{R}$ such that $\left|r_{R}\right|=k$. So, there are $1+2 C_{n}^{1}+2^{2} C_{n}^{2}+\cdots+2^{n} C_{n}^{n}$ pairs of $\left(r_{P}, r_{R}\right)$ such that $r_{P} \subseteq r_{R}$. Thus,

$$
\begin{aligned}
\tau_{n}(D) & =\frac{1}{\left|\mathscr{M}_{n}\right|} \sum\left\{\tau_{\mathbf{M}}(D) \mid \mathbf{M} \in \mathscr{M}_{n}\right\} \\
& =\frac{1}{2^{2 n}} \sum\left\{1+2 C_{n}^{1}+2^{2} C_{n}^{2}+\cdots+2^{n} C_{n}^{n}\right\} \\
& =\frac{3^{n}}{2^{2 n}}=\left(\frac{3}{4}\right)^{n} .
\end{aligned}
$$

In conclusion, $\vec{\tau}(D)=\left(\frac{3}{4}, \frac{9}{16}, \cdots,\left(\frac{3}{4}\right)^{n}, \cdots\right)$.

Remark 2. (i) According to example 3 and example 4, with the increase of the $n$ value, $\tau_{n}(A)$ and $\tau_{n}(D)$ are decreasing, $\tau_{n}(B)$ remains the constant $\frac{1}{2}$ unchanged, and $\tau_{n}(C)$ is increasing. Furthermore, the $n$-validity degrees of $A, B, C, D$ tend to the constants $\frac{1}{2}, \frac{1}{2}, 1$ and 0 respectively.

(ii) Although the average validity degrees of all these formulae are all equal to $\frac{1}{2}$, from their validity degree vectors, the truth degrees of $A$ and $C$ are both larger than $B^{\prime} s$ whatever the value of $n$ is. Furthermore, when the value of $n$ is sufficiently large, the order of the $n$-validity degrees of $A, B, C, D$ is: $\tau_{n}(D) \leqslant \tau_{n}(A) \leqslant \tau_{n}(B) \leqslant \tau_{n}(C)$.

(iii) From the above examples, it seems that the validity degree vectors of first-order formulae can give us more information about the order of their truth degrees, and moreover tell us the changing trends of truth degrees of formulae with the increase of the cardinality of the domain of the finite interpretation. Actually, it is true. The vector form can describe the truth degrees of formulae more intuitively and comprehensively, and the value is an average of all of the relative satisfiability degrees in all finite interpretations in $\mathscr{M}_{n}$ so that it can characterize the truth degrees of formulae more precisely and delicately.

In the following, we will study some basic properties, and denote that

$$
\overrightarrow{0}=(0,0, \cdots, 0, \cdots), \overrightarrow{1}=(1,1, \cdots, 1, \cdots),
$$

$$
\vec{\alpha}=(\alpha, \alpha, \cdots, \alpha, \cdots), \vec{\beta}=(\beta, \beta, \cdots, \beta, \cdots) .
$$

Note that the form of infinite dimensional vector of $\vec{\tau}(A)$ can present the validity degree more intuitively and comprehensively, but it still can not tell us the validity degree of a formula in any infinite interpretation. In fact, we have the following theorem.

Theorem 3. Suppose $A \in \mathscr{F}$.

(i) $\vec{\tau}(A)=\overrightarrow{1}$ iff $\tau_{\mathbf{M}}(A)=1$ for every interpretation $\mathbf{M} \in \mathscr{M}_{f}$;

(ii) $\vec{\tau}(A)=\overrightarrow{0}$ iff $\tau_{\mathbf{M}}(A)=0$ for every interpretation $\mathbf{M} \in \mathscr{M}_{f}$.

The following theorem is the immediate conclusion of proposition 2 and theorem 3 .

Theorem 4. Suppose $A \in \mathscr{F}$, then $\bar{\tau}(A)=1$ iff $\vec{\tau}(A)=\overrightarrow{1}$, and $\bar{\tau}(A)=0$ iff $\vec{\tau}(A)=\overrightarrow{0}$.

Theorem 4 tells us that, for the validity degree vectors and the average validity degrees of formulae, the judgement to the relative "best" and "worst" formulae is consistent in the sense of the finite interpretations. So the validity degree vectors divide all the first-order formulae more exactly than the average validity degrees do, but they are compatible.

Some basic properties are studied in the following proposition.

Proposition 5. Let $A, B \in \mathscr{F}, n=1,2, \cdots$.

(i) $0 \leqslant \tau_{n}(A) \leqslant 1$, and $\overrightarrow{0} \leqslant \vec{\tau}(A) \leqslant \overrightarrow{1}$;

(ii) $\tau_{n}(A)+\tau_{n}(\neg A)=1$, and $\vec{\tau}(A)+\vec{\tau}(\neg A)=\overrightarrow{1}$;

(iii) If $A \sim B$, then $\tau_{n}(A)=\tau_{n}(B)$, and $\vec{\tau}(A)=\vec{\tau}(B)$;

(iv) If $A$ is logically valid, then $\tau_{n}(A)=1$, and $\vec{\tau}(A)=\overrightarrow{1}$; Similarly, if $A$ is a contradiction, then $\tau_{n}(A)=0$, and $\vec{\tau}(A)=\overrightarrow{0}$.

Remark 3. (i) According to remark 1 and proposition 5(i), it is clear that $\tau_{n}(\neg A)=\frac{1}{2}(n=1,2, \cdots)$ holds for every atomic formula $A$, that is, $\vec{\tau}(\neg A)=$ $\left(\frac{1}{2}, \frac{1}{2}, \cdots, \frac{1}{2}, \cdots\right)$.

(ii) It's worthy to note that the converses of the proposition 5(iv) is false, that is, $A$ may not be logically efficient even if it is true w.r.t. every finite interpretation. In fact, the formula $A$ given by (6) is a counterexample ${ }^{[44]}$. But, from proposition 1 , we can easily prove that, for a big class of first-order formulae, the property of the validity degree vector $\overrightarrow{1}$ is equivalent to be logically valid. 
Theorem 6. Suppose that $A \in \mathscr{F}, A$ is a prenex normal form. If A contains no quantifiers, or the quantifiers in A are all universal or all existential, then

(i) $A$ is logically valid if and only if $\vec{\tau}(A)=\overrightarrow{1}$;

(ii) $A$ is a contradiction if and only if $\vec{\tau}(A)=\overrightarrow{0}$.

Proposition 7. Let $A, B, C \in \mathscr{F}$.

(i) $\vec{\tau}(A \vee B)+\vec{\tau}(A \wedge B)=\vec{\tau}(A)+\vec{\tau}(B)$;

(ii) If $\vec{\tau}(A) \geqslant \vec{\alpha}, \vec{\tau}(A \rightarrow B) \geqslant \vec{\beta}$, then $\vec{\tau}(B) \geqslant \vec{\alpha}+$ $\vec{\beta}-\overrightarrow{1}$;

(iii) If $\vec{\tau}(A \rightarrow B) \geqslant \vec{\alpha}, \vec{\tau}(B \rightarrow C) \geqslant \vec{\beta}$, then $\vec{\tau}(A \rightarrow$ $C) \geqslant \vec{\alpha}+\vec{\beta}-\overrightarrow{1}$.

Proof. (i) According to (12) and (14), we only need to prove that

$$
\tau_{n}(A \vee B)+\tau_{n}(A \wedge B)=\tau_{n}(A)+\tau_{n}(B)
$$

holds for every positive integer $n$. Because the definition of $\tau_{n}(A)$ given by the equation (11) is actually obtained by taking the average of all values of $\tau_{\mathbf{M}}(A)$ where $\mathbf{M} \in \mathscr{M}_{n}$, it suffices to prove the following equality:

$$
\tau_{\mathbf{M}}(A \vee B)+\tau_{\mathbf{M}}(A \wedge B)=\tau_{\mathbf{M}}(A)+\tau_{\mathbf{M}}(B), \forall \mathbf{M} \in \mathscr{M}_{f}
$$

In fact, according to (7),

$$
\begin{aligned}
\tau_{\mathbf{M}}(A \vee B)= & \mu_{\mathbf{M}}\left([A \vee B]_{\mathbf{M}}\right) \\
= & \mu_{\mathbf{M}}\left([A]_{\mathbf{M}} \cup[B]_{\mathbf{M}}\right) \\
= & \mu_{\mathbf{M}}\left([A]_{\mathbf{M}}\right)+\mu_{\mathbf{M}}\left([B]_{\mathbf{M}}\right) \\
& -\mu_{\mathbf{M}}\left([A]_{\mathbf{M}} \wedge[B]_{\mathbf{M}}\right) \\
= & \mu_{\mathbf{M}}\left([A]_{\mathbf{M}}\right)+\mu_{\mathbf{M}}\left([B]_{\mathbf{M}}\right) \\
& -\mu_{\mathbf{M}}\left([A \wedge B]_{\mathbf{M}}\right) \\
= & \tau_{\mathbf{M}}(A)+\tau_{\mathbf{M}}(B)-\tau_{\mathbf{M}}(A \wedge B) .
\end{aligned}
$$

(ii) We only need to prove the corresponding conclusion about $\tau_{n}$, that is, if $\tau_{n}(A) \geqslant \alpha$ and $\tau_{n}(A \rightarrow$ $B) \geqslant \beta$, then $\tau_{n}(B) \geqslant \alpha+\beta-1$. From the equation (16),

$$
\begin{aligned}
\tau_{n}(A \rightarrow B) & =\tau_{n}(\neg A \vee B) \\
& =\tau_{n}(\neg A)+\tau_{n}(B)-\tau_{n}(\neg A \wedge B) \\
& =1-\tau_{n}(A)+\tau_{n}(B)-\tau_{n}(\neg A \wedge B) .
\end{aligned}
$$

So, it follows from the precondition that

$$
\begin{aligned}
\tau_{n}(B) & =\tau_{n}(A)+\tau_{n}(\neg A \wedge B)+\tau_{n}(A \rightarrow B)-1 \\
& \geqslant \tau_{n}(A)+\tau_{n}(A \rightarrow B)-1 \\
& \geqslant \alpha+\beta-1 .
\end{aligned}
$$

(iii) Similarly, it suffices to prove that $\tau_{n}(A \rightarrow$ $C) \geqslant \alpha+\beta-1$ if $\tau_{n}(A \rightarrow B) \geqslant \alpha$ and $\tau_{n}(B \rightarrow C) \geqslant$ $\beta$. According to the equation (16) and the precondition,

$$
\begin{aligned}
\tau_{n}(A \rightarrow B)= & \tau_{n}(\neg A \vee B) \\
= & \tau_{n}[(\neg A \vee C) \vee(B \vee \neg C)] \\
= & \tau_{n}(\neg A \vee B)+\tau_{n}(\neg B \vee C) \\
& -\tau_{n}[(\neg A \vee B) \wedge(\neg B \vee C)] \\
= & \tau_{n}(A \rightarrow B)+\tau_{n}(B \rightarrow C) \\
& -\tau_{n}[(\neg A \vee B) \wedge(\neg B \vee C)] \\
\geqslant & \alpha+\beta-1 .
\end{aligned}
$$

From Proposition 7, it can be easily proved that the validity degree vectors of formulae can preserve the logical relation between formulae, that is, we have the following conclusion.

Theorem 8. Suppose $A, B, C \in \mathscr{F}$.

(i) If $\vec{\tau}(A \rightarrow B)=\overrightarrow{1}$, then $\vec{\tau}(A) \leqslant \vec{\tau}(B)$; Especially, if $\vec{\tau}(A)=\vec{\tau}(A \rightarrow B)=\overrightarrow{1}$, then $\vec{\tau}(B)=1$.

(ii) If $\vec{\tau}(B \rightarrow C)=\overrightarrow{1}$, then $\vec{\tau}(A \rightarrow B) \leqslant \vec{\tau}(A \rightarrow$ $C)$; Especially, if $\vec{\tau}(A \rightarrow B)=\vec{\tau}(B \rightarrow C)=\overrightarrow{1}$, then $\vec{\tau}(A \rightarrow C)=\overrightarrow{1}$.

After the use of the rule of generalization, the change in the validity degree vectors of formulae is as follows:

Proposition 9. Suppose $A \in \mathscr{F}, n=1,2, \cdots$.

(i) If $\tau_{n}(A)=1$, then $\tau_{n}((\forall x) A)=1$. Therefore, if $\vec{\tau}(A)=\overrightarrow{1}$, then $\vec{\tau}((\forall x) A)=\overrightarrow{1}$.

(ii) $\tau_{n}((\forall x) A) \leqslant \tau_{n}(A)$, and therefore $\vec{\tau}((\forall x) A) \leqslant$ $\vec{\tau}(A)$.

\section{Consistency theorem}

According to remark 1 and remark 3 , the $n$-validity degrees of all atomic formulae and their negations are $\frac{1}{2}$ and irrelevant to the value of $n$. Then, are there other formulae whose $n$-validity degrees are irrelevant to the value of $n$ ? In this section, a consistency theorem tells us that, for a big class of first-order formulae, their $n$-validity degrees are all equal for every $n$, and furthermore are equal to the validity degrees of the corresponding propositions.

In the following, we will denote $\mathscr{F}_{0}$ the set of all quantifier-free formulae, $\mathscr{F}_{1}$ denote the set of all 
quantifier-free formulae without any repeated predicate symbols and terms, and $\left.\left(r_{P}\right)_{P}\right|_{A}$ and $\left.\left(m_{c}\right)_{c}\right|_{A}$ respectively denote the interpretations for the predicate symbols and individual constants in the formula $A$. Suppose $A, B \in \mathscr{F}$, and $\mathbf{M}=\left(M_{n},\left.\left(r_{P}\right)_{P}\right|_{A} \cup\right.$ $\left.\left.\left(r_{P}\right)_{P}\right|_{B},\left.\left.\left(m_{c}\right)_{c}\right|_{A} \cup\left(m_{c}\right)_{c}\right|_{B}\right) \in \mathscr{M}_{n}$, then denote $\mathbf{M}_{A}=$ $\left(M_{n},\left.\left(r_{P}\right)_{P}\right|_{A},\left.\left(m_{c}\right)_{c}\right|_{A}\right), \mathbf{M}_{B}=\left(M_{n},\left.\left(r_{P}\right)_{P}\right|_{B},\left.\left(m_{c}\right)_{c}\right|_{B}\right)$.

Proposition 10. If $A \wedge B \in \mathscr{F}_{1}$, then $\tau_{n}(A \wedge B)=$ $\tau_{n}(A) \tau_{n}(B)$

Proof. Since $v(c)=m_{c}$ is already fixed for every individual constant $c$, the valuation $v \in \Omega_{\mathbf{M}}$ is completely determined by its restriction $v \mid W$ on the set $W=\left\{x_{1}, x_{2}, \cdots\right\}$ of variables. Assuming that variables in $A$ and $B$ are respectively $x_{1}, \cdots, x_{r}$ and $y_{1}, \cdots, y_{s}$, the valuation $v \in \Omega_{\mathbf{M}}$ is determined by the array $\left(v\left(x_{1}\right), \cdots, v\left(x_{r}\right), v\left(y_{1}\right), \cdots, v\left(y_{s}\right)\right) \in$ $M^{r+s}$. Because $A \wedge B \in \mathscr{F}_{1}$, that is, there are no common predicate symbols, variables and individual constants in $A$ and $B,\left(v\left(x_{1}\right), \cdots, v\left(x_{r}\right)\right)$ satisfying $A$ is independent to $\left(v\left(y_{1}\right), \cdots, v\left(r_{s}\right)\right)$ satisfying $B$. Moreover, it's clear that $\|A\|_{\mathbf{M}, v}=\|A\|_{\mathbf{M}_{A}, v}$ and $\|B\|_{\mathbf{M}, v}=\|B\|_{\mathbf{M}_{B}, v}$. Therefore, it follows from (7) that

$$
\begin{aligned}
\tau_{\mathbf{M}}(A \wedge B)= & \mu_{\mathbf{M}}\left(\left\{\vec{v}=\left(v\left(x_{1}\right), \cdots, v\left(x_{r}\right), v\left(y_{1}\right),\right.\right.\right. \\
& \left.\cdots, v\left(y_{s}\right)\right) \in X_{M} \mid v \in \Omega_{\mathbf{M}}, \\
& \left.\left.\|A \wedge B\|_{\mathbf{M}, v}=1\right\}\right) \\
= & \mu_{\mathbf{M}}\left(\left\{\vec{v}=\left(v\left(x_{1}\right), \cdots, v\left(x_{r}\right), v\left(y_{1}\right),\right.\right.\right. \\
& \left.\cdots, v\left(y_{s}\right)\right) \in M_{n}^{r+s} \mid v \in \Omega_{\mathbf{M}} \\
& \left.\left.\|A\|_{\mathbf{M}, v}=1, \text { and }\|B\|_{\mathbf{M}, v}=1\right\}\right) \\
= & \mu_{\mathbf{M}}\left(\left\{\left(v\left(x_{1}\right), \cdots, v\left(x_{r}\right)\right) \in M^{r}\right.\right. \\
& \left(v\left(y_{1}\right), \cdots, v\left(y_{s}\right)\right) \in M_{n}^{s} \mid v \in \Omega_{\mathbf{M}}, \\
& \left.\left.\|A\|_{\mathbf{M}, v}=1, \text { and }\|B\|_{\mathbf{M}, v}=1\right\}\right) \\
= & \mu_{\mathbf{M}}\left(\left\{\left(v\left(x_{1}\right), \cdots, v\left(x_{r}\right)\right) \in M_{n}^{r} \mid\right.\right. \\
& \left.\left.v \in \Omega_{\mathbf{M}},\|A\|_{\mathbf{M}, v}=1\right\}\right) \times \\
& \mu_{\mathbf{M}}\left(\left\{\left(v\left(y_{1}\right), \cdots, v\left(y_{s}\right)\right) \in M_{n}^{s} \mid\right.\right. \\
& \left.\left.v \in \Omega_{\mathbf{M}},\|B\|_{\mathbf{M}, v}=1\right\}\right) \\
= & \mu_{\mathbf{M}_{A}}\left(\left\{\left(v\left(x_{1}\right), \cdots, v\left(x_{r}\right)\right) \in M_{n}^{r} \mid\right.\right. \\
& \left.\left.v \in \Omega_{\mathbf{M}_{A}},\|A\|_{\mathbf{M}_{A}, v}=1\right\}\right) \times \\
& \mu_{\mathbf{M}_{B}}\left(\left\{\left(v\left(y_{1}\right), \cdots, v\left(y_{s}\right)\right) \in M_{n}^{s} \mid\right.\right. \\
& \left.\left.v \in \Omega_{\mathbf{M}_{B}},\|B\|_{\mathbf{M}_{B}, v}=1\right\}\right) \\
= & \tau_{\mathbf{M}_{A}}(A) \tau_{\mathbf{M}_{B}}(B) .
\end{aligned}
$$

On the other hand, we only consider the firstorder language in $A$ and $B$. If we denote $\mathscr{M}_{n}^{A}$ and $\mathscr{M}_{n}^{B}$ the set of all the interpretations $\mathbf{M} \in \mathscr{M}_{n}$ for predicate symbols and individual constants respectively in $A$ and $B$, then it's clear that $\left|\mathscr{M}_{n}\right|=\left|\mathscr{M}_{n}^{A}\right|$. $\left|\mathscr{M}_{n}^{B}\right|$.

Therefore, it follows from (11) that

$$
\begin{aligned}
\tau_{n}(A \wedge B)= & \frac{1}{\left|\mathscr{M}_{n}\right|} \sum\left\{\tau_{\mathbf{M}}(A \wedge B) \mid \mathbf{M} \in \mathscr{M}_{n}\right\} \\
= & \frac{1}{\left|\mathscr{M}_{n}^{A}\right| \cdot\left|\mathscr{M}_{n}^{B}\right|} \sum\left\{\tau_{\mathbf{M}_{A}}(A) \tau_{\mathbf{M}_{B}}(B) \mid\right. \\
& \left.\mathbf{M}_{A} \in \mathscr{M}_{n}^{A}, \mathbf{M}_{B} \in \mathscr{M}_{n}^{B}\right\}
\end{aligned}
$$

It can be proved that $\sum_{i, j} a_{i} b_{j}=\sum_{i} a_{i} \sum_{j} b_{j}$, so

$$
\begin{aligned}
\tau_{n}(A \wedge B) & =\frac{\sum\left\{\tau_{\mathbf{M}_{A}}(A) \mid \mathbf{M}_{A} \in \mathscr{M}_{n}^{A}\right\} \sum\left\{\tau_{\mathbf{M}_{B}}(B) \mid \mathbf{M}_{B} \in \mathscr{M}_{n}^{B}\right\}}{\left|\mathscr{M}_{n}^{A}\right| \cdot\left|\mathscr{M}_{n}^{B}\right|} \\
& =\tau_{n}(A) \tau_{n}(B) .
\end{aligned}
$$

Proposition 11. Suppose that $E=Q_{1} \wedge Q_{2} \wedge$ $\cdots \wedge Q_{m} \in \mathscr{F}_{1}$, where $Q_{i}=p_{i}$ or $Q_{i}=\neg p_{i}(i=$ $1,2, \cdots, m), p_{i}$ is an atomic formula, then $\tau_{n}(E)=$ $\frac{1}{2^{m}}$.

Proof. According to remark 1 and remark 3, $\tau_{n}\left(Q_{i}\right)=\frac{1}{2}(i=1,2, \cdots, m)$. Therefore it follows from proposition 10 that $\tau_{n}(E)=\frac{1}{2^{m}}$.

Proposition 12. Let $F \in \mathscr{F}_{1}$, then $\bar{\tau}(F)=\frac{1}{2}$. Proof. Suppose $p_{1}, \cdots, p_{m}$ are the atomic formulae appearing in $F$ and $P_{i}$ is the predicate symbol appearing in $p_{i}(i=1,2, \cdots, m)$. For each finite interpretation $\mathbf{M}$ and each valuation $v \in \Omega_{\mathbf{M}}$, it's clear that $\left\|p_{i}\right\|_{\mathbf{M}, v}(i=1,2, \cdots, m)$ is independent of each other. By substituting $x_{1}, \cdots, x_{m}$ for $p_{1}, \cdots, p_{m}$ respectively, and interpreting the logical connectives $\neg$ and $\rightarrow$ as the corresponding operations $\neg$ and $\rightarrow$ defined in (1), we then obtain an $m$-ary function $\bar{F}:\{0,1\}^{m} \rightarrow\{0,1\}$. According to (1), it is not difficult verify that the function $\bar{F}$ is a surjection, that is, $\bar{F}^{-1}(0) \neq \emptyset$ and $\bar{F}^{-1}(1) \neq \emptyset$. Assuming that $\left(x_{1}^{\prime}, \cdots, x_{m}^{\prime}\right) \in \bar{F}^{-1}(1)$, construct the finite interpretation $\mathbf{M}_{1}=\left(M_{1},\left(r_{P}\right)_{P},\left(m_{c}\right)_{c}\right) \in \mathscr{M}_{f}$ such that $r_{P_{i}}=$ $M_{1}^{x_{i}^{\prime}}$, where $M_{1}^{1}=M_{1}$ and $M_{1}^{0}=\emptyset$, then $\|F\|_{\mathbf{M}_{1}, v}=1$ holds for any valuation $v$ in $\Omega_{\mathbf{M}_{1}}$, and furthermore $\tau_{\mathbf{M}_{1}}(F)=1$. Similarly, we can construct a finite interpretation $\mathbf{M}_{2}$ such that $\tau_{\mathbf{M}_{1}}(F)=0$. According to (8), we can obtain that $\bar{\tau}(F)=\frac{1}{2}$.

According to theorem 4 and proposition 5(iv), it is easy to verify the following proposition. 
Proposition 13. There are no logical valid formulae or contradictions in $\mathscr{F}_{1}$.

Lemma 14. ${ }^{[6]}$ Every formula in $\mathscr{F}_{0}$ is provably (or equivalently, logically) equivalent to a formula in disjunctive (conjunctive) normal form.

Theorem 15. Suppose that $A \in \mathscr{F}_{1}$, then there exist two positive integers $m, k$ such that $\tau_{n}(A)=\frac{k}{2^{m}}$.

Proof. Since $A \in \mathscr{F}_{1} \subset \mathscr{F}_{0}$, it follows from Lemma

14 that $A$ is logically equivalent to a formula in disjunctive normal form. Suppose that $p_{1}, \cdots, p_{m}$ are the atomic formulae appearing in $A$, and they contain no common predicate symbols or terms. Due to proposition 13, $A$ is not a contradiction. Then $A$ has an equivalent form as follows:

$$
\begin{gathered}
A \sim A^{\prime}=A_{1}^{\prime} \vee \cdots \vee A_{k}^{\prime}, \\
A_{1}^{\prime}=Q_{11} \wedge \cdots \wedge Q_{1 m}, \cdots, A_{k}^{\prime}=Q_{k 1} \wedge \cdots \wedge Q_{k m},
\end{gathered}
$$

where $Q_{i j}=p_{j}$ or $Q_{i j}=\neg p_{j}(i=1, \cdots, k ; j=$ $1, \cdots, m)$. To prove the theorem, it suffices to prove that $\tau_{n}\left(A^{\prime}\right)=\frac{k}{2^{m}}$.

Note that there exists $j$ such that $Q_{i_{1}, j}=\neg Q_{i_{2}, j}$ or $Q_{i_{2}, j}=\neg Q_{i_{1}, j}$ holds whenever $i_{1} \neq i_{2}$. So $A_{i_{1}}^{\prime} \wedge A_{i_{2}}^{\prime}$ is a contradiction whenever $i_{1} \neq i_{2}$. Due to (16),

$$
\tau_{n}\left(A^{\prime}\right)=\tau_{n}\left(A_{1}^{\prime}\right)+\cdots+\tau_{n}\left(A_{k}^{\prime}\right) .
$$

Moreover, it's clear that $A_{i}^{\prime} \in \mathscr{F}_{1}(i=1, \cdots, k)$ because they contain no common predicate symbols or terms. So it follows from proposition 11 that $\tau_{n}\left(A_{i}^{\prime}\right)=\frac{1}{2^{m}}(i=1,2, \cdots, k)$. So $\tau_{n}\left(A^{\prime}\right)=\frac{k}{2^{m}}$.

Substitute the proposition $p_{i}^{0}$ for the atomic firstorder formula $p_{i}$ in $A$ and $A^{\prime}$, then we can obtain the corresponding propositions $A^{0}$ and $A^{\prime 0}$. Since $A$ is logically equivalent to $A^{\prime}$ in first-order logic, $A^{0}$ and $A^{\prime} 0$ are logically equivalent to each other in two-valued propositional logic. On account of the computation of truth degrees in two-valued logic(see ref.[6]), it is easy to verify that $\tau\left(A^{\prime 0}\right)=\frac{k}{2^{m}}$, and moreover $\tau\left(A^{0}\right)=\frac{k}{2^{m}}$. Therefore we have the following consistency theorem.

Theorem 16. (consistency theorem) Let $A \in \mathscr{F}_{1}$, then

$$
\tau_{n}(A)=\tau\left(A^{0}\right), n=1,2, \cdots .
$$

And moreover,

$$
\vec{\tau}(A)=\left(\tau\left(A^{0}\right), \cdots, \tau\left(A^{0}\right), \cdots .\right)
$$

Example 5. Let $E=P(x, y) \rightarrow Q(c, z, d)$. Substitute $P(x, y)$ and $Q(c, z, c)$ respectively for the atomic propositions $p$ and $q$, we can obtain the corresponding proposition $E^{0}=p \rightarrow q$. In two-valued propositional logic, it is easy to verify that $\tau\left(E^{0}\right)=\frac{3}{4}$. Hence from theorem 16, we have

$$
\vec{\tau}(E)=\left(\frac{3}{4}, \cdots, \frac{3}{4}, \cdots\right) .
$$

Let it be noted that it would be rather troublesome if we use a general way to calculate the value of $\tau_{n}(E)$ starting from the definition given by (11). Fortunately, the consistency theorem can make the calculations much more convenient by calculating $\tau\left(E^{0}\right)$ in two-valued propositional logic, without considering any interpretations or valuations on variables and individual constants. In twovalued propositional logic, $v(p \rightarrow q)=0 \neq 1$ holds if and only if $(v(p), v(q))=(1,0)$. Because there are totally 4 possible valuations on $\{p, q\}, \tau\left(E^{0}\right)=$ $1-\frac{1}{4}=\frac{3}{4}$. So we can immediately conclude that $\tau_{n}(E)=\frac{3}{4}$.

\section{Conclusions}

The present paper proposes a new kind of representation for the validity degrees of first-order formulae, that is, the validity degree vectors of formulae, which is much more comprehensive and intuitive to describe the validity degree of a first-order formula than the average validity degrees of formulae. Moreover, it is proved that the validity degree vectors can preserve the logical relation between formulae. Furthermore, there are a consistency theorem between the $n$-validity degrees of first-order formulae and the validity degrees of corresponding propositions. In the following, we will try to define the similarity degree and the distance between two first-order formulae, study the distribution of the validity degree vectors of formulae in vector space $[0,1]^{\infty}$, and develop the theory of approximate reasoning in two-valued predicate logic. 


\section{Acknowledgments}

This work is supported by National Science Foundation of China (Grant No.61175055), Sichuan Key Technology Research and Development Program (Grant No.2011FZ0051).

\section{References}

1. A.G. Hamilton, "Logic For Mathematicians", Cambridge University Press, London,(1978).

2. J.B. Rosser, A.R. Turequtte, "Many-Valued Logics", North-Holland, Amsterdam, (1952).

3. P. Hájek, "Metamathematics of Fuzzy Logic", Kluwer Academic Publishers, London, (1998).

4. M. De Glas, "Knowledge representation in a fuzzy setting", Technical Report 89/48, Universite Paris VI, Laforia, (1989).

5. J. Pavelka, ”On fuzzy logic I, II, III”, Zeitschr. f. Math. Logik u. Grundlagen d. Math., 25, 45-52, 119-134, 447-464 (1979).

6. Guo-Jun Wang, Hong-Jun Zhou,'Introduction to Mathematical Logic and Resolution Principle(Second Ed.)",Science Press, Beijing,(2009)

7. Y. Xu, D. Ruan, K. Qin, J. Liu, 'Lattice-Valued LogicAn Alternative Approach to Treat Fuzziness and Incomparability", Springer, NewYork, (2003).

8. G.J. Wang, L. Fu, J.S. Song, "Theory of truth degrees of propositions in two-valued logic", Sci. China A, 45(9) 1106-1116 (2002).

9. Wang G. J., Leung Y., ’Integrated semantic and logic metric spaces", Fuzzy Sets Syst, 136, 71-91 (2003).

10. Li B.J., Wang G.J., "Theory of truth degrees of formulas in Łukasiewicz n-valued propositional logic and a limit theorem", Sci China Ser F, 48(6), 727-736 (2005).

11. Wang G. J., Qian G. S., Dang C. Y., "Theory unified approximate reasoning in $L^{*}$ propositional calculus system and $K^{*}$ predicate calculus system", $S c i$ China Ser F-Inf Sci, 48(1), 1-14 (2005).

12. H.J. Zhou, G.J. Wang, "A new theory consistency index based on deduction theorems in several logic systems", Fuzzy Sets Syst., 157, 427-443 (2006).

13. Han C., Wang G. J., Xu W., "A 16-classification partition of formula set $\mathrm{F}(\mathrm{S})$ in revised Kleene System $\bar{W}$,, Soft Comput, 10(2), 115-118 (2006).

14. Li J., Wang G. J., "Theory of truth degrees of propositions in the logic system $L_{n}^{*}$, Sci China Ser F-Inf Sci, 49(4) 471-483 (2006).

15. Zhou H. J., Wang G. J., Zhou W., "Consistency degrees of theories and methods of graded reasoning in $n$-valued $R_{0}$-logic (NM-Logic)", Int J Approx Reason, 43, 117-132 (2006).
16. Wang G. J., Hui X. J., "Randomization of classical inference patterns and its application", Sci China Ser F-Inf Sci, 50(6), 867-877 (2007).

17. Wang G. J., Hui X.J., Song J.S., "The $R_{0}$-type fuzzy logic metric space and an algorithm for solving fuzzy modus ponens", Comput Math Appl, 55, 1974-1987 (2008).

18. Wang G. J., Duan Q. L., 'Theory of (n) truth degrees of formulas in modal logic and a consistency theorem”, Sci China Ser F, 52, 70-83 (2009).

19. Hongbo $\mathrm{Wu}$, "The generalized truth degree of quantitative logic in the logic system $\mathscr{L}_{n}^{*}(n$-valued NMlogic system)", Computers and Mathematics with Applications, 59, 2587-2596 (2010).

20. Zhou HongJun,Wang GuoJun,'Borel probabilistic and quantitative logic",Science China:Information Sciences,54(9):1843-1854(2011)

21. J. Li, Y. Zhou, "A Quantitative Method of n-valued Göel Propositional Logic", Procedia Environmental Sciences, 12, 583-589 (2012).

22. Xiaofeng Ye,Weibing Zuo,"The Randomization of R0-logic With Six-valued Nonlinear Valuation Set",Physics Procedia,25,2146-2151(2012).

23. Zhang Xiao-Hong,Dai Jian-Hua,'Rough implication operation and residuated based fuzzy logic system",The Journal of China Universities of Posts and Telecommunications,20(Suppl. 1),109-112(2013)

24. Wu HongBo,'The theory of $\Gamma$-truth degrees of formulas and limit theorem in Lukasiewicz propositional logic", Science China:Information Science, 44(12), 1542-1559 (2014)

25. Yanhong She,'On the rough consistency measures of logic theories and approximate reasoning in rough logic",International Journal of Approximate Reasoning,55,486-499(2014).

26. Hui Xiaojing, "Quantified axiomatic extension systems of SBLs based on truth value", Sci. China:Information Science, 44(7),900-911(2014).(in Chinese)

27. Jian Ren Zhou, Hong Bo Wu,'The Characteristics of Logic Functions Determined by $R_{0}$-Implication Operator", Acta Mathematic Sinica(Chinese Series), 57(2),235-248(2014).

28. Guojun Wang, Hongjun Zhou, "Quantitative logic", Information Sciences, 179, 226-247 (2009).

29. Cheng Li,Hua-Wen Liu,Guo-Jun Wang,'Correction and improvement on several results in quantitative logic",Information Sciences, 278,555-558(2014).

30. W. Bandler, L.J. Kohout, "Semantics of implication operators and fuzzy relational products", Internat. J. ManCMachine Stud., 12, 89-116 (1980).

31. W. Bandler, L.J. Kohout, "The interrelations of the principal fuzzy logical operators", in: M.M. Gupta, A. Kandel,W. Bandler, J.B. Kiszka (Eds.), ”Approximate Reasoning in Expert Systems", North-Holland, Ams- 
terdam, 767-780 (1985).

32. W. Bandler, L.J. Kohout, "The use of checklist paradigm in inference systems", in: C.V. Negoita, H. Prade (Eds.), "Fuzzy Logic in Knowledge Engineering”, Verlag TÜV Rheinland, Köln, 95-111 (1986).

33. P. Hjek, L.J. Kohout, "Fuzzy implications and generalized quantifiers", Internat. J. Uncertainty, Fuzziness and Knowledge Based Systems, 4(3) 225-233 (1996).

34. L.J. Kohout,W. Bandler, "Interval-valued systems for approximate reasoning based on the checklist paradigm", in: P.Wang, Paul (Eds.), "Advances in Fuzzy Theory and Technology(vol. 1)", Bookwrights Press, Durham, N.C, 167-193 (1993).

35. L.J. Kohout, E. Kim, "Characterization of interval fuzzy logic systems of connectives by group transformations", Reliable Comput, 10 299C334 (2004).

36. G.J. Wang, X.Y. Qin, X.N. Zhou, "An intrinsic fuzzy set on the universe of discourse of predicate formulas", Fuzzy Sets Syst, 157, 3145-3158 (2006).

37. Qin X.Y., Liu J., Xu Yang, Chen S.W., Liu Y., 'Theory of approximate reasoning in two-valued predicate logic based on the quasi-truth degrees", Journal of Donghua University, 29(1), 23-27 ( 2012).
38. Qin X.Y.,Liu Y.,Xu Y.,'The degree of the quasisimilarity and the pseudo-metric between predicate formulae",Proceedings of the 2nd International Conference on Quantitative Logic and Soft Computing, 273-283(2010).

39. Qin X.Y.,Liu Y.,Xu Y.,Zhang J.F.,'On the quasisimilarity degree between first-order formulae",Proceedings of 9th International FLINS Conference on Foundations and Applications of Computational Intelligence, 547-552(2010).

40. Wang G.J., "Axiomatic theory of truth degree for a class of first-order formulas and its application", $S c i$ China-Inf Sci, 42(5), 648-662 (2012).

41. Blackburn P, Rijke M, Venema Y, "Modal Logic", Cambridge University Press, London, (2001).

42. D.W. Barnes, J.M. Mack, "An Algebraic Introduction to Mathematical Logic", Springer, NewYork, (1975).

43. P.R. Halmos, "Measure Theory", Van Nostrand, New York, (1950).

44. Y. Ang, "Ideas and Methods of mathematical logic", Fudan University Press, Shanghai, (1991)(in Chinese). 